\title{
Control domain wall motion by different depth trenches in submicron permalloy wires
}

\author{
Cheng-Yi Wu ${ }^{1 *}$ and Shiow-Kang Yen ${ }^{2}$ \\ 1 Department of Materials Engineering, National Chung Hsing University, Taichung, 40227, Taiwan; \\ d100066007@mail.nchu.edu.tw \\ 2 Department of Materials Engineering, National Chung Hsing University, Taichung, 40227, Taiwan; \\ skyen@dragon.nchu.edu.tw \\ * Correspondence: d100066007@mail.nchu.edu.tw; Tel.: +886-4-2284-0500 ext609
}

\begin{abstract}
Domain walls were studied for permalloy wires with different depth trenches (500 nm wide, $30 \mathrm{~nm}$ thick, $7.5 \mu \mathrm{m}$ long, the depth of the trench was $0,4,5,8,10,12$ and $15 \mathrm{~nm}$ ). Permalloy ( $\mathrm{Ni}_{80} \mathrm{Fe}_{20}$ ) wires were fabricated by electron beam lithography and Ar ion milling. When the depth of trench is smaller than $12 \mathrm{~nm}$, the switching field $\left(\mathrm{H}_{\mathrm{s}}\right)$ is increasing with the deeper trench. However, the depth of trench is bigger than half depth of thickness, the $\mathrm{H}_{\mathrm{s}}$ is decreasing with the deeper trench. We believed that $\mathrm{H}_{\mathrm{s}}$ increased as the trench depth increased was because the magnetic diploes force increased between the magnetic poles on two sides of the trench. The domains of the wires were divided by the trenches and the domain walls were pinned on the trenches these were confirmed by magnetic force microscopy images.
\end{abstract}

Keywords: domain walls, magnetic force microscope; magnetoresistance; permalloy wire.

\section{Introduction}

Recently, magnetoresistance (MR) and magnetic force microscopy (MFM) measurements have been widely used to study electric transport properties and the magnetization process magnetic in submicron metallic wires [1,2]. Both MR and MFM have attracted much attention because of the influence of domain nucleation and annihilation during the magnetization process. And the Magnetization process is controlled by the motion of the domain wall (DW), so it is significant to manipulate the DW motion during the magnetization process. There are many methods to trap the DW[3], they make some expertise such as notch that trap the injected DW. The notch was used to decrease the cross-section, and this way will also diminish the DW energy, thus the DW pinning was trapped in the notch. However, we consider the modern microlithography and fabrication in semiconducting industry, the ratio of notch/(width wire) come to a limit. In this paper, we use the trench to trap the injected domain wall. The distinct from the notch, the accurate of trench can be controlled by the mature technique, and an error on the depth of the trench can be fabricated to smaller than $1 \mathrm{~nm}$.

\section{Materials and Methods}

We designed a special stripe magnetic wire as schematically illustrated in Figure 1. One end of the stripe magnetic wire is connected to a diamond-shaped pad which acts as a DW injector [4], and the other end is sharply pointed to prevent the nucleation of a DW from this end [5]. Then the different depth trenches were fabricated at the middle of the stripe. The diamond-shaped wire of $30 \mathrm{~nm}$-thick $\mathrm{Ni}_{80} \mathrm{Fe}_{20}$ were deposited onto thermal $\mathrm{SiO}_{2}$ substrates at Ar pressure $10^{-3}$ torr by DC magnetron sputtering after a base pressure less than $5 \times 10^{-7}$ torr and then patterned using electron-beam lithography (EBL). The film was then patterned into different depth trenches using e-beam lithography followed by low-current argon-ion milling, and the depth of the trench is 4, 5, 8, 10, 12 and $15 \mathrm{~nm}$, respectively. Subsequently, four $\mathrm{Cu} / \mathrm{Au}$ contact leads were deposited on top of the $\mathrm{Ni}_{80} \mathrm{Fe}_{20}$ wire by a lithographical lift-off process [6]. We report the MR and MFM studies on permalloy 
submicron-wires with fixed diamond-shape elements in different configurations. Magnetorestance measurements were made by a standard four-probe technique at room-temperature and external magnetic field was applied in sample plane. The domain wall schematic figure structure was monitored by magnetic force microscopy imaging (MFM).

Figure 2(a) shows a scanning electron microscope (SEM) image of permalloy device. Domain will be nucleated in the diamond pad and then allows the injection of domain wall into the stripe when the magnetic field is applied along the stripe. The MFM image (Figure 2(b)) confirmed the magnetic structure, a closure domain nucleated in the diamond-shaped and there was magnetic flux (black and white) just beside the trench. The contrast (black and white) increased as the increasing of the depth. This means that the magnetizations in the depth of the trench and the domain- shape align to the same direction.

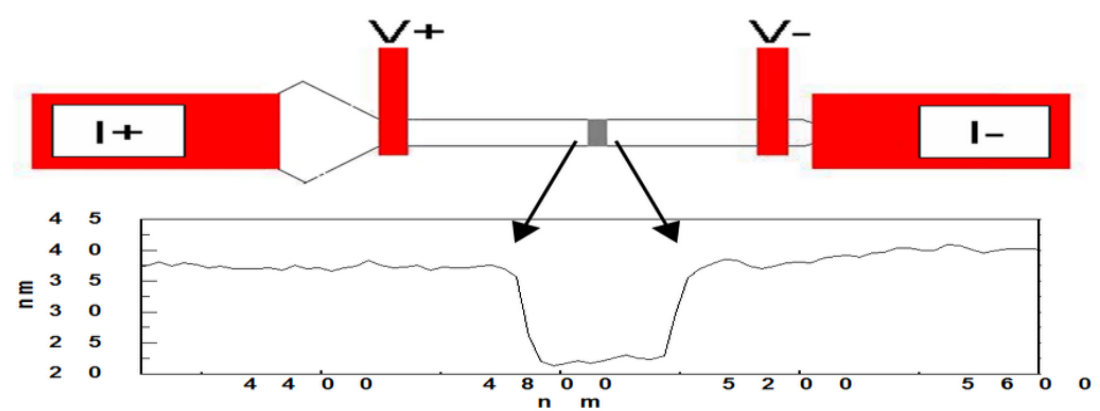

Figure 1. Schematic illustration of the diamond-shape series wire, and the AFM profile of trench.

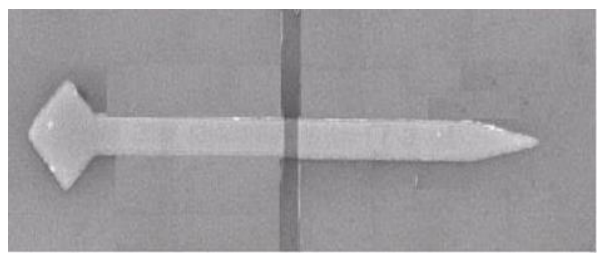

(a)

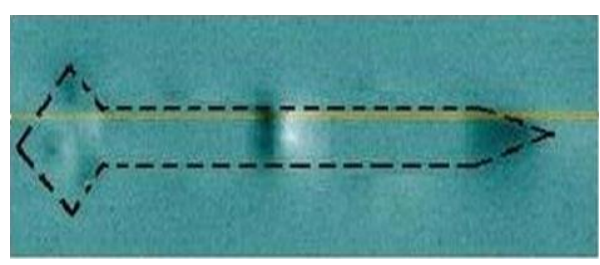

(b)

Figure 2. (a). SEM picture of the diamond-stripe series wire and (b) The MFM images of the device. The dash line is the guide line for the wire.

\section{Results and Discussion}

Longitudinal MR was measured to investigate the DW pinning force via the depth of the trench. Figure 3(a) (d). shows the MR loop of the wire with the different depth of the trench. The wire without trench is known that the swift motion of DW, so we did not observe the variation of MR loop in the longitudinal direction. And from the depth of $10 \mathrm{~nm}$, we get the switching field $\left(\mathrm{H}_{\mathrm{s}}=\right.$ $106 \pm 4$ Oe). It can tell us the anisotropic MR (AMR) effect during magnetization reversal process. The MR curve have gradual drop when the external magnetic field was swept through from positive saturation field, it represents that the nucleation process in the trench.

In order to study the $\mathrm{H}_{\mathrm{s}}$ (switching field) with different depth of the trench, we have arranged the date in Figure 4. When the depth of trench is smaller than $12 \mathrm{~nm}$, the $\mathrm{H}_{\mathrm{s}}$ increased with the deeper of trench. This phenomenon was caused by the stronger DW pinning force, because the thin of the trench, the dipole interaction in the trench will become more and more. However, the depth of trench is bigger than $12 \mathrm{~nm}$, the $\mathrm{H}_{\mathrm{s}}$ decreased with the deeper trench, it tells us the thinner depth of trench separate the stripe magnetic wire like two shorter wires. So, the $\mathrm{H}_{s}$ decreased when the trench is thinner than the certain depth. 


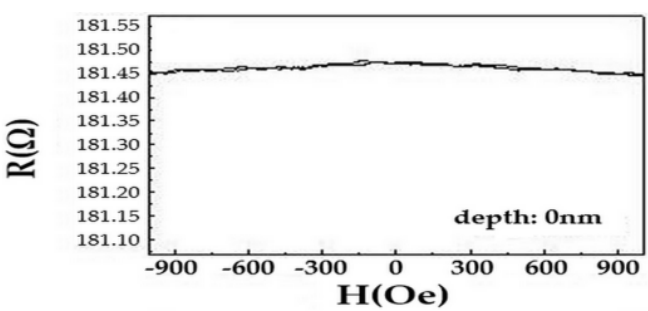

(a)

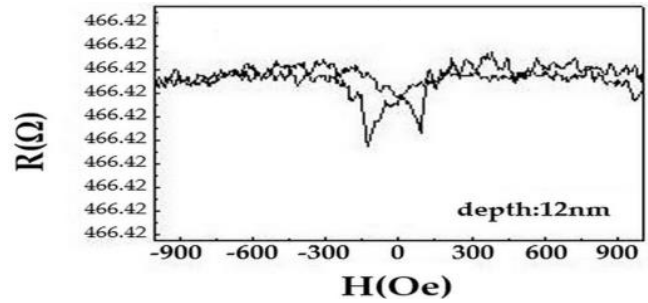

(c)

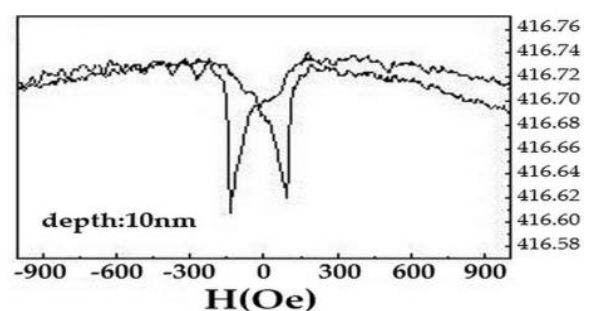

(b)

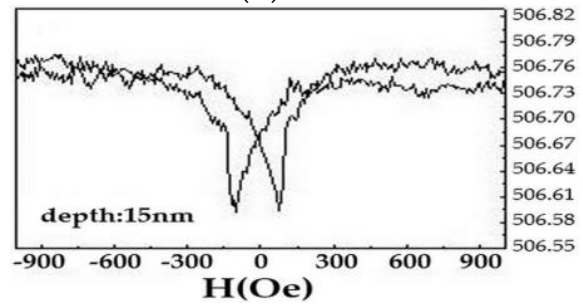

(d)

Figure 3. MR curves measured at room temperature for the longitudinal directions.(a) (d). The depth of the trench is o, 10, 12 and $15 \mathrm{~nm}$, respectively.

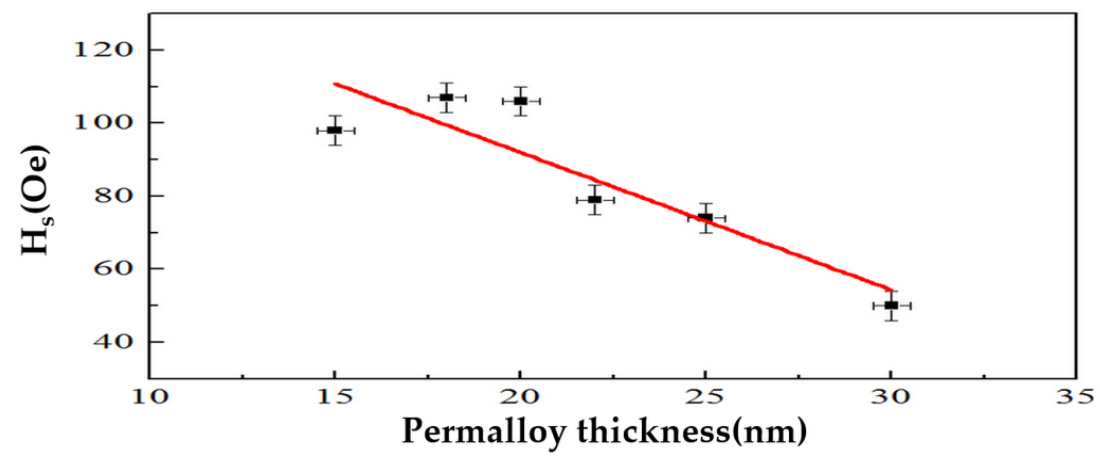

Figure 4. The relations between the switching field and different depth of the trench.

\section{Conclusions}

In this study, we succeed in using MFM to explain the magnetizations in the depth of the trench. Besides, the result of the depth in the trench would affect the $\mathrm{H}_{\mathrm{s}}$, and when the trench getting more than half depth of thickness, the Hs decrease instead.

Funding: This research received no external funding.

Acknowledgments: The authors would like to thank Dr. K. W. Cheng for his assistance during the experimental measurements.

Conflicts of Interest: The authors declare no conflict of interest.

\section{References}

1. Y. Ishikuro, M. Kawaguchi, Y.C. Lau, Y. Nakatani and M. Hayashi, Applied Physics Express, 2018; 11, 7.

2. X Z Chen, H Liu, L F Yin, H Chen, C Song and F Pan, Journal of Physics D: Applied Physics, 2017; 50, 50.

3. M. Vázquez, G. A. Basheed, G. Infante, and Rafael P. Del Real , Phys. Rev. Lett., 2012; 108, 037201.

4. T. Phung, A. Pushp, L. Thomas and C. Rettner, etc. Nano Lett., 2015; 15, 835.

5. S. Lepadatu, H. Saarikoski, and R. Beacham, etc. Scientific Reports, 2017; 7, 1640.

6. L. Wang, R.Y. Zhang, and X.J. Wang, etc., Materials Research Express, 2018; 5, 1. 